\title{
Photoluminescence Characterisation of High Current Density Resonant Tunnelling Diodes for Terahertz Applications
}

\author{
Kristof J. P. JACOBS ${ }^{\dagger \text { a) }}$, Benjamin J. STEVENS ${ }^{\dagger}$, and Richard A. HOGG ${ }^{\dagger}$, Nonmembers
}

\begin{abstract}
SUMMARY High structural perfection, wafer uniformity, and reproducibility are key parameters for high-volume, low cost manufacture of resonant tunnelling diode (RTD) terahertz (THz) devices. Low-cost, rapid, and non-destructive techniques are required for the development of such devices. In this paper, we report photoluminescence (PL) spectroscopy as a non-destructive characterisation technique for high current density InGaAs/AlAs/InP RTD structures grown by metal-organic vapour phase epitaxy (MOVPE) for THz applications. By using a PL line scanning technique across the edge of the sample, we identify characteristic luminescence from the quantum well (QW) and the undoped $/ \mathrm{n}^{+}$InGaAs layers. By using the Moss-Burstein effect, we are able to measure the free-electron concentration of the emitter/collector and contact layers in the RTD structure. Whilst the $\mathrm{n}^{+}$InGaAs luminescence provides information on the doping concentration, information on the alloy composition and compositional variation is extracted from the InGaAs buffer layer. The QW luminescence provides information on the average well width and provides a monitor of the structural perfection with regard to interface and alloy disorder.

key words: resonant tunnelling diode, high-speed electronics, terahertz, photoluminescence
\end{abstract}

\section{Introduction}

The terahertz $(\mathrm{THz})$ frequency band spans the region of the electromagnetic spectrum between the microwaves and the infrared light $(100 \mathrm{GHz}-10 \mathrm{THz})$. Optical and electronic devices have been developed to operate in the $\mathrm{THz}$ spectrum, but often suffer from a low output power. Due to the technological challenges to develop efficient emitters in the $\mathrm{THz}$ region, this spectrum is also known as the "terahertz gap" [1]. Solid-state electronic devices operating in this spectrum are mainly limited by the carrier transit-time, whereas optical devices require low energy band-to-band transitions. Recent optical $\mathrm{THz}$ devices include the p-type Ge laser [2], THz quantum cascade laser (QCL) [3], THz wave parametric amplifier [4], and the uni-travelling carrier photodiode (UTC-PD) [5]. On the electron device side, devices such as the tunnel injection transit time negative resistance (TUNNETT) diode [6], Gunn diode [7], high-electronmobility transistor (HEMT) [8], and resonant tunnelling diode (RTD) [9] are being studied. $\mathrm{THz}$ waves will provide new capabilities for applications in various fields, including ultra-broadband wireless communications [10], spectroscopic sensing and imaging [11], [12], and high-resolution radar [13]. The lower frequency band of $275-3000 \mathrm{GHz}$ has

Manuscript received July 25, 2015.

Manuscript revised October 5, 2015.

${ }^{\dagger}$ The authors are with the Department of Electronic \& Electrical Engineering, University of Sheffield, United Kingdom.

a)E-mail: elp11kjj@gmail.com

DOI: 10.1587/transele.E99.C.181 not been allocated yet for specific uses, and offers very high wireless data, which matches the current bandwidths of fibre optic communications. In order to allow systems to satisfy Edholm's Law of bandwidth in future, new wireless technologies are needed which can support tens of Gbit/s [14]. In 2011, Mukai et al. demonstrated wireless data transmission at $1.5 \mathrm{Gbit} / \mathrm{s}$ using a RTD oscillator in the $300 \mathrm{GHz}$ band [15]. Compact and coherent solid-state sources are key components for such applications.

To date, the RTD is recognised as the fastest electronic device, with fundamental oscillation up to $1.86 \mathrm{THz}$ reported at room temperature [16]. The crystal growth of these devices is particularly challenging as the current-voltage (IV) characteristic is highly sensitive to the epitaxial layers parameters (i.e. thickness, composition, and doping). The barrier thickness is one of the main parameters for the RTD as the peak current density increases exponentially with reducing thickness [17].

Figure 1 (a) shows a schematic of the typical I-V characteristic from a $3.3 \mu \mathrm{m}^{2}$ device and the inset shows a scanning electron microscope (SEM) image of the device and the maximum RTD output power formula. To maximise the output power of the device, large voltage spans $(\Delta \mathrm{V})$ and current spans $(\Delta \mathrm{I})$ are required in the negative differential conductance (NDC) region, as depicted in Fig. 1 [18]. A low peak voltage and high peak-to-valley current ratio (PVCR) are also key requirements to minimise wasteful power consumption of the device. Figure 1 (b) pictographically shows the typical $0.35 \mathrm{THz}$ RTD emitter. The RTD is monolith-

(a)
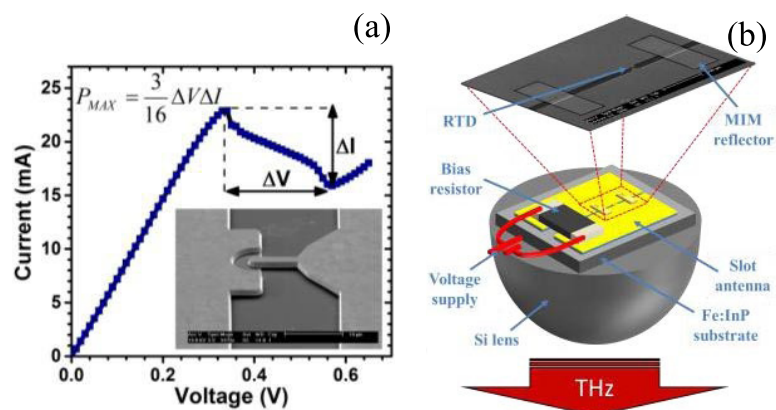

Fig. 1 (a) I-V characteristic from the typical $\sim 3.3 \mu \mathrm{m}^{2} \mathrm{RTD}$. The inset shows a scanning electron microscope (SEM) image of the device and maximum RTD output power formula with $\Delta \mathrm{V}$ and $\Delta \mathrm{I}$ defined as the voltage and current spans in the NDC region. (b) Schematic of the RTD THz emitter operating at $0.35 \mathrm{THz}$. The SEM image is zoomed-in on the RTD and slot antenna. 
ically integrated with a slot antenna using metal-insulatormetal (MIM) reflectors. The chip is placed upon a hyperhemispherical lens to couple emission out of the substrate and has an integrated surface-mount stabilisation resistor to inhibit parasitic oscillations over the bias supply lines. The RTD oscillates when it is biased in the NDC region as the negative conductance negates the resistive and radiative losses in the circuit.

The RTD THz emitter is expected to offer great potential for a wide variety of applications, it being compact, coherent, and integratable with other functional electron/optical devices [19], [20]. Room temperature operation of the device is also clearly seen as an advantage for the large and ever-growing market in portable electronic devices.

Low-cost mass-manufacture of such devices requires good wafer-to-wafer reproducibility and wafer-scale uniformity of the device characteristics. The RTD is not commercialised yet, as the required level of reproducibility of the RTD for high-volume low cost manufacture (typically less than 5\% [21]) has not been fully demonstrated. In 2011, Sugiyama et al. demonstrated the potential of MOVPE as a production technique for RTD devices, reporting high uniformity and reproducibility [22]. A peak current variation of $7.9 \%$ was obtained over 3" wafers, which corresponds to on-wafer AlAs barrier thickness uniformity of $0.03 \mathrm{~nm}$. Mass production of RTDs will require sub-ML thickness control of the QW to minimise effects such as interface roughness scattering which can considerably degrade the RTD performance. Transmission electron microscopy (TEM), secondary ion mass spectrometry (SIMS), electrochemical capacitance-voltage (eCV) profiling [23], X-ray diffractometry (XRD), and photoluminescence (PL) spectroscopy are techniques typically applied for the material analysis of such structures. In particular, XRD and PL are highly favored production environment characterisation tools as they are non-destructive. Sugiyama et al. previously reported on the abruptness of the double barrier heterointerfaces, qualified using the high-frequency Pendellosung fringes on the XRD pattern, and the measurement of the total thickness of the double barrier structure (well + barriers) measured from the XRD intensity beat caused by diffraction from the double barrier structure [22]. However, characterising the structural parameters of such structures remains problematic as the double barrier structure of a high current density RTD is typically very thin $(<10 \mathrm{~nm})$ and is not periodic.

The quantum well $(\mathrm{QW})$ perfection and uniformity, and doping uniformity of the contact and emitter/collector layers over the wafer are important parameters for a low device variability and high yield. Monolayer (ML) fluctuations of the thin tunnelling barriers are expected to be of great importance in these structures [24]. As the tunneling current is also largely dependent on the doping concentration in the emitter/collector regions, a uniform doping concentration is desired for low device variability with regard to uniform I-V characteristics and contact resistances over the wafer [22]. Even with the steady improvement in epitaxial growth techniques, there are still challenges ahead for manufacturability [25].

As efforts toward solving the problems associated with the low-cost manufacture of tunnel devices is still limited, there is a need for low-cost, rapid, and non-destructive material characterisation methods to provide rapid feedback to the epitaxial process. Low cost manufacture requires good wafer-to-wafer reproducibility and uniformity for a high yield without the requirement of pre-testing each device. In 2002, Hayden et al. reported on an ex-situ recalibration method using a calibration structure to rapidly analyse the epitaxy using XRD and eCV to correct for actual layer thicknesses and dopant concentrations [26]. Using this technique, little variation in the electrical characteristics of tunnel devices was measured from wafers grown several months apart. However, this procedure requires frequent recalibration, which comes at the expense of sacrificial epitaxial wafers. Being able to perform the materials analysis on the actual device epitaxy is clearly seen as an advantage, allowing monitoring of wafer production, and reducing set-up costs which are required for calibration.

PL spectroscopy is a powerful, sensitive, nondestructive, and contactless characterisation technique to probe the electronic structure of semiconductor materials [27]. This technique is typically applied for the evaluation of performance of optical devices on the wafer scale [28], but can be equally well applied to probe epitaxy of electronic devices with a direct band-gap structure. However, the optical properties of high current density InGaAs/AlAs/InP RTDs remain so far relatively unexplored [29]-[31].

In this work, we report on PL characterisation of high current density RTDs for $\mathrm{THz}$ applications. We explore the PL method as a characterisation tool to enable routes for device optimisation and enable volume manufacture. The optical properties of high current density $\left(\sim 700 \mathrm{kA} / \mathrm{cm}^{2}\right)$ InGaAs/AlAs/InP RTDs grown by MOVPE are investigated in detail. Temperature dependent PL is carried out to investigate localised states in the RTD structure, and a line scan technique across the edge of the sample is used to explore the origin of the emission in the structure. From the measured spectra, we are able to non-destructively measure the doping concentrations of the emitter/collector and contact layers in the device by using the Moss-Burstein (MB) effect. The MB effect is a shift in optical band-gap with increasing carrier density. It results from the Pauli exclusion principle, with the Fermi level located in the conduction/valence band, for n-type/p-type material, and fundamental transitions to already filled states being forbidden. Our results are confirmed using eCV profiling. eCV profiling is a technique used to determine the free-electron concentration by analysing the capacitance-voltage $(\mathrm{C}-\mathrm{V})$ behavior of the developed region of a reverse-biased Schottky barrier which is formed between the electrolyte and the surface of the sample [23]. Depth profiling is achieved by electrolytically etching between $\mathrm{C}-\mathrm{V}$ measurements. 

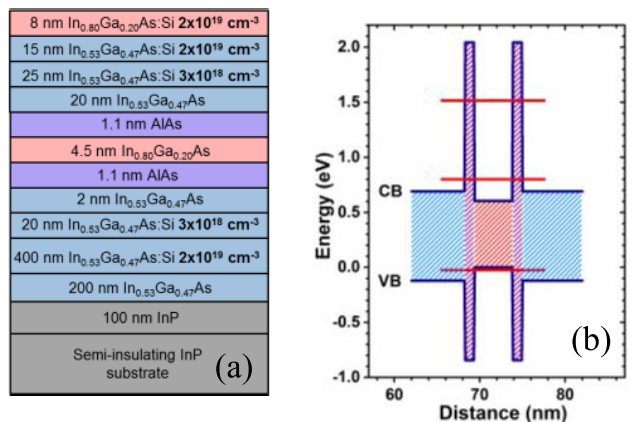

Fig. 2 (a) Schematic of the RTD layer structure (b) band structure of the double barrier RTD at $15 \mathrm{~K}$. CB and VB are defined as the conduction band and valence band, respectively.

We go on to investigate the well width dependent luminescence characteristics of the RTD structure. Using low temperature PL, we are able to probe the interface roughness of the barrier interfaces non-destructively, thereby providing valuable information from a manufacturing perspective and enabling routes for future device optimisation.

\section{Epitaxial Material and Band Structure}

The InP/InGaAs/AlAs RTD structures were grown in a vertical Thomas Swan $6 \times 2^{\prime \prime}$ close-coupled shower head MOVPE reactor on (100) semi-insulating InP:Fe substrates with a $0.07^{\circ}$ offcut. Details about the growth process are reported elsewhere [31].

Figure 2 schematically shows (a) the layer structure of the $4.5 \mathrm{~nm}$ QW RTD, and (b) the low temperature band structure. The horizontal red lines indicate the first and second electron states and first heavy-hole state. The band alignments are determined by the model-solid theory and the resonant energy levels are modelled with the transfer matrix method. The epitaxy of the RTD structure consists of a $100 \mathrm{~nm}$ InP buffer layer, followed by $200 \mathrm{~nm}$ $\mathrm{In}_{0.53} \mathrm{Ga}_{0.47} \mathrm{As}$, and $400 \mathrm{~nm}$ highly n-doped $\operatorname{In}_{0.53} \mathrm{Ga}_{0.47} \mathrm{As}$ $\left(2 \times 10^{19} \mathrm{~cm}^{-3} \mathrm{Si}\right)$ to serve as the lower contact. A $20 \mathrm{~nm}$ n-doped $\mathrm{In}_{0.53} \mathrm{Ga}_{0.47} \mathrm{As}\left(3 \times 10^{18} \mathrm{~cm}^{-3} \mathrm{Si}\right)$ emitter layer is subsequently grown, followed by a $2 \mathrm{~nm} \mathrm{In}_{0.53} \mathrm{Ga}_{0.47} \mathrm{As}$ spacer layer. An $\operatorname{In}_{0.80} \mathrm{Ga}_{0.20}$ As quantum well is formed between two $1.1 \mathrm{~nm}$ AlAs barriers. On the collector side, a $20 \mathrm{~nm} \operatorname{In}_{0.53} \mathrm{Ga}_{0.47}$ As spacer layer is grown with a $25 \mathrm{~nm} \mathrm{In}{ }_{0.53} \mathrm{Ga}_{0.47}$ As $\left(3 \times 10^{18} \mathrm{~cm}^{-3} \mathrm{Si}\right)$ collector layer. The epitaxy is terminated with $15 \mathrm{~nm}$ n-doped $\operatorname{In}_{0.53} \mathrm{Ga}_{0.47} \mathrm{As}$ $\left(2 \times 10^{19} \mathrm{~cm}^{-3} \mathrm{Si}\right)$, and $8 \mathrm{~nm} \mathrm{In} \operatorname{In}_{0.80} \mathrm{Ga}_{0.20}$ As $\left(2 \times 10^{19} \mathrm{~cm}^{-3} \mathrm{Si}\right)$ to enhance the formation of a low-resistance ohmic contact at the collector side. RTD structures with QW thicknesses of 3.0, 3.5, 4.0, and $4.5 \mathrm{~nm}$ were grown.

\section{Low Temperature Photoluminescence Spectroscopy}

Low temperature PL spectroscopy has been widely used to investigate the carrier transport and localised states in quantum-well structures. Skolnick et al. previously stud-

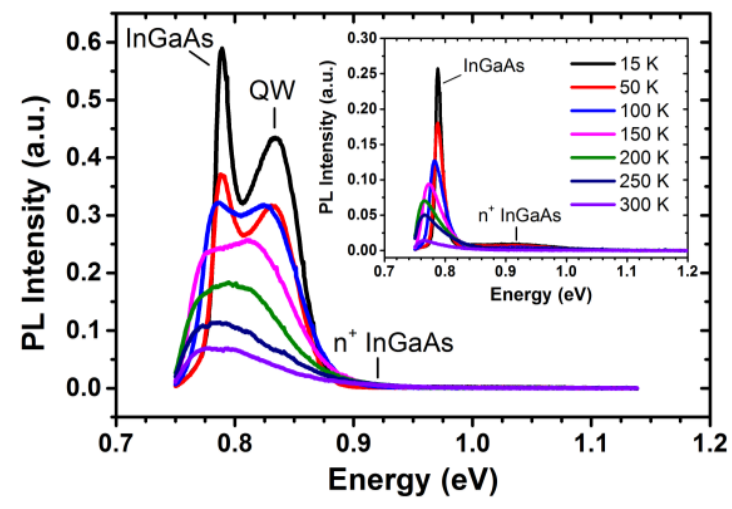

Fig. 3 Temperature dependent PL spectra of the RTD structure without and with (inset) the QW etched off, from 15 to $300 \mathrm{~K}$.

ied the excitation mechanisms which lead to PL in QWs of $\mathrm{GaAs} / \mathrm{AlGaAs}$ double barrier resonant tunnelling structures (RTS) under bias. The transport properties and charge accumulation of GaAs/AlGaAs RTS were also investigated by a number of workers using low temperature PL [32]-[34] and time-resolved PL spectroscopy [35]. In 1991, Tews et al. reported a luminescence study of MOVPE grown GaAs/AlGAs RTD structures using low temperature PL [36]. In 1996, Shen et al. reported a PL study of InAs/InGaAs/AlAs strained single quantum well structures [30].

In this work, temperature dependent PL characterisation was performed over a temperature range from 15 to $300 \mathrm{~K}$ in a closed cycle helium (He) cryostat. Operating at low temperatures has the advantage of not only minimising the effects of many thermally activated non-radiative processes (e.g. surface recombination velocity) and thermal line broadening which can obscure important information, but it also ensures that nearly all carriers are placed in their ground state which reduces the complexity of the analysis from interactions between ground state and excited states [37]. A frequency doubled neodymium doped yttrium vanadium oxide $\left(\mathrm{Nd}: \mathrm{YVO}_{4}\right)$ laser at $532 \mathrm{~nm}$ was mechanically chopped at $20 \mathrm{~Hz}$ to excite the samples. The PL measurements were performed with an excitation power of $15 \mathrm{~mW}$ focused on the sample to a spot of $\sim 75 \mathrm{um}$ in diameter. The luminescence was dispersed by a double grating Bentham DMc150 monochromator and detected by an InGaAs transimpedance amplified photodetector coupled using the conventional lock-in technique. The PL spectra are shown uncorrected as the spectral response of the InGaAs detector does not vary by more than $20 \%$ over the full region of interest $(0.75-1.0 \mathrm{eV})$.

Figure 3 shows temperature dependent PL spectra of the $4.5 \mathrm{~nm}$ QW RTD structure (shown schematically in Fig. 2 (a)) from 15 to $300 \mathrm{~K}$. The inset of Fig. 3 shows temperature dependent PL spectra of the same sample with the QW etched off using eCV as an accurate selective etch. More details about this technique are reported elsewhere [31]. When the sample is cooled below $50 \mathrm{~K}$, thermal line broadening effects are reduced, and the broad 


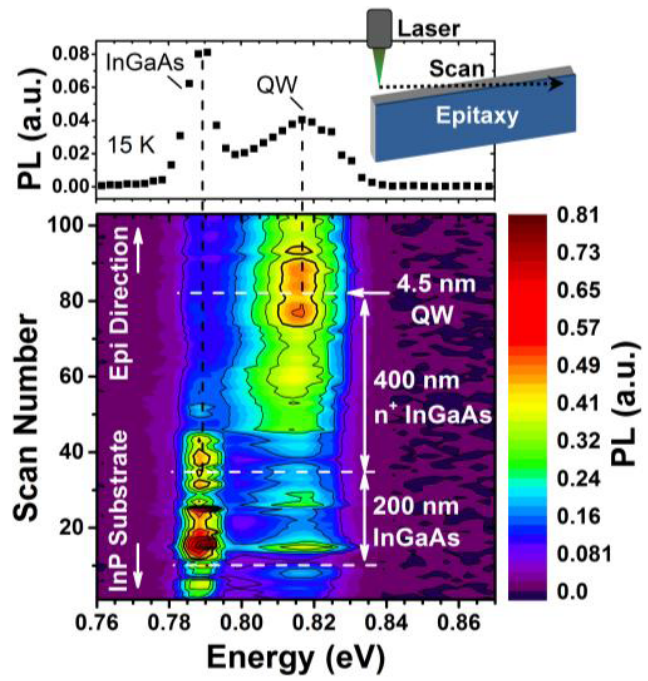

Fig. 4 PL measured at $15 \mathrm{~K}$ as a function of energy measured along the scan direction. The inset pictographically shows the PL line scan technique performed across the edge of the sample.

emission resolves into two distinctive emission features at $789 \mathrm{meV}$ and $834 \mathrm{meV}$. A broad and weak emission at $\sim 0.92 \mathrm{eV}$, which is more visible in the inset, is also measured. The emission at $834 \mathrm{meV}$ is assigned to the $\mathrm{QW}$ as it is no longer observed when the QW is etched off.

To further explore the origin of the low temperature PL features, a low temperature PL line scan was carried out across the edge of the sample. Figure 4 shows the PL intensity as a function of energy for 103 scans measured across the edge of the sample in the epitaxial direction with a 10 micron step size, as indicated. The technique, schematically illustrated in the inset of Fig. 4, allows a partially quantitative PL map with higher resolution than can be obtained using standard positioning of the stages due to the very shallow angle used of less than 1 degree. Due to the finite excitation spot size, the PL emission from the $4.5 \mathrm{~nm}$ QW is spread across multiple spectra. Assuming a spread of \pm 10 scans, we are able to assign the emission at $789 \mathrm{meV}$ to the undoped InGaAs buffer layer. The measured spatial separation of the QW and InGaAs emission is in very good agreement with the $400 \mathrm{~nm}$ of the lower $\mathrm{n}^{+}$InGaAs contact layer. As no emission is observed at the band-gap energy of InGaAs for the undoped InGaAs spacer layers, it is suggested that photogenerated carriers in this region tunnel rapidly into the QW, and the electrostatic potential profile associated with the doped RTD spatially separates the carriers, preventing radiative recombination within the undoped spacer layers closest to the QW. The broad emission at $\sim 0.92 \mathrm{eV}$ is in good agreement with expected luminescence from $\mathrm{n}^{+}$ InGaAs [38].

\subsection{Luminescence in High Current Density RTDs}

Figure 5 pictographically shows the electronic band structure of (a) the undoped InGaAs buffer layer (b) $n^{+}$InGaAs emitter/collector/contact layer, and (c) the double barrier
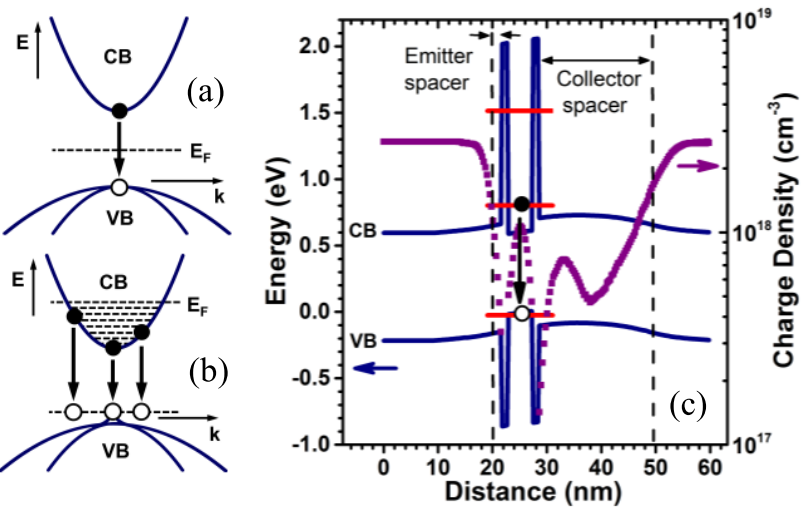

Fig. 5 Low temperature band structures of (a) InGaAs buffer layer (b) $\mathrm{n}^{+}$ InGaAs emitter/collector/contact layers, and (c) double barrier RTD structure. CB is defined as the conduction band, VB as the valence band, and $\mathrm{E}_{\mathrm{F}}$ as the Fermi energy.

RTD structure. The arrows represent the ground state recombination between photo-excited electron and hole pairs at low temperatures. For undoped InGaAs, shown in Fig. 5 (a), the probablility of recombination is greatest for states near the band edges. For $\mathrm{n}^{+}$InGaAs in Fig. 5 (b), the optical band-gap increases due to the MB effect. The spectral linewidth broadens with increasing free-electron concentration as electrons below the Fermi level (energetic position dependent on the free-electron concentration) recombine with the deeper acceptor-line tail states [39]. Whilst the undoped $\mathrm{InGaAs}$ buffer layer is buried $400 \mathrm{~nm}$ below the $\mathrm{n}^{+}$ InGaAs layer of the lower contact layer, a stronger PL signal is measured. The lower radiative recombination efficiency of the $\mathrm{n}^{+} \mathrm{InGaAs}$ layer is associated with increased Auger recombination [40]. This is in strong agreement with observations made by Ahrenkiel and co-workers, showing that Auger recombination becomes the dominant recombination mechanism for doping densities greater than $5 \times 10^{18} \mathrm{~cm}^{-3}$ in InGaAs [41].

Figure 5 (c) shows the calculated electronic band structure and charge density profile of the RTD using NEMO5, a simulation tool based on the non-equilibrium Green's functions (NEGFs) formalism [42]. A Hartree model is used to solve the quantum charge self-consistently with the electrostatic potential. The electrostatic potential is raised on the collector side with respect to the emitter, due to the asymmetric spacer layers. The first and second electron states and first heavy-hole state are indicated by horizontal red lines. An electron density of $1 \times 10^{18} \mathrm{~cm}^{-3}$ is determined within the well with no bias applied to the structure. This quantum well charge originates from the diffusion of carriers from the dopant atoms of the highly doped emitter and collector layers. Luminescence originates from the $\mathrm{QW}$, as these doping diffused electrons in the QW recombine with captured photo-generated holes in the compressively strained $\mathrm{In}_{0.80} \mathrm{Ga}_{0.20}$ As well. As compressive strain in the QW splits the degenerate heavy-hole (HH) and light-hole (LH) bands, the $\mathrm{HH}$ transition is measured due to the suppression of the LH transition. 


\subsection{Photoluminescence for the Measurement of Doping}

Whilst SIMS and eCV are useful techniques for the analysis of impurities and free-carrier concentration, both techniques are destructive, relying on the removal of material. PL is well regarded as a powerful, non-destructive, highly sensitive technique to evaluate impurity concentrations due to the high energy resolution. This method has previously been applied to determine low concentrations of impurities. Tajima applied low temperature PL at liquid He temperatures to measure the impurity concentration of phosphorus and boron in silicon by measuring the intensity ratio of the PL spectral lines [43]. The intensity ratio between intrinsic and extrinsic components is used in the PL spectra to reflect the doping density by eliminating differential nonradiative effects. This technique works very well at low impurity concentrations $\left(\mathrm{n}<10^{15} \mathrm{~cm}^{-3}\right)$ but becomes impractical for high impurity concentrations as the intrinsic peak becomes obscured by the impurity peak. A different method was demonstrated by De-Sheng et al. for the analysis of heavily doped n-type GaAs [39]. The energy position of the Fermi level and the energy half-width on the emission band was measured in degenerate $n$-type GaAs allowing the free-electron concentration to be determined by low temperature PL measurements. We apply a similar technique to non-destructively measure the free-electron concentration in InGaAs but instead of measuring the energy position at the Fermi level we measure the energy at $-20 \mathrm{~dB}$ intensity at the high energy doping tail. This avoids the interference of the InGaAs and QW emission with the measurement and the difficulty in assigning the peak energy of the broad $\mathrm{n}^{+}$ InGaAs emission. Using this technique, we were able to investigate the doping uniformity of the $\mathrm{n}^{+}$InGaAs contact and emitter/collector layers using two separate InGaAs teststructures [31]. In this work, we apply the same technique but instead of using dedicated test-structures, we measure the free-electron concentration in the actual RTD structure.

Figure 6 shows the low temperature PL spectrum of the RTD on a logarithmic scale. The top right inset shows the required calibration curve for the PL method to determine the free-electron concentration. More details of this calibration are reported elsewhere [31]. The energies at $-20 \mathrm{~dB}$ of both high energy tails are indicated by arrows in Fig. 6 . Using the calibration curve, free-electron concentrations of $6 \times 10^{18} \mathrm{~cm}^{-3}$ and $3 \times 10^{19} \mathrm{~cm}^{-3}$ are determined for the emitter/collector and contact layers, respectively. The bottom left inset in Fig. 6 shows the measured free-electron concentration as a function of depth in the RTD structure using $\mathrm{eCV}$ profiling. The measured free-electron concentration using our PL method is in good agreement with the measured free-electron concentrations for the emitter/collector $\left(\mathrm{n}=5 \times 10^{18} \mathrm{~cm}^{-3}\right)$ and contact layers $\left(\mathrm{n}=3 \times 10^{19} \mathrm{~cm}^{-3}\right)$. We note that whilst this technique is very useful to evaluate impurity concentrations $>10^{17} \mathrm{~cm}^{-3}$, this technique will become more difficult to apply for impurity concentrations $<10^{17} \mathrm{~cm}^{-3}$, due to the small PL energy shift at low

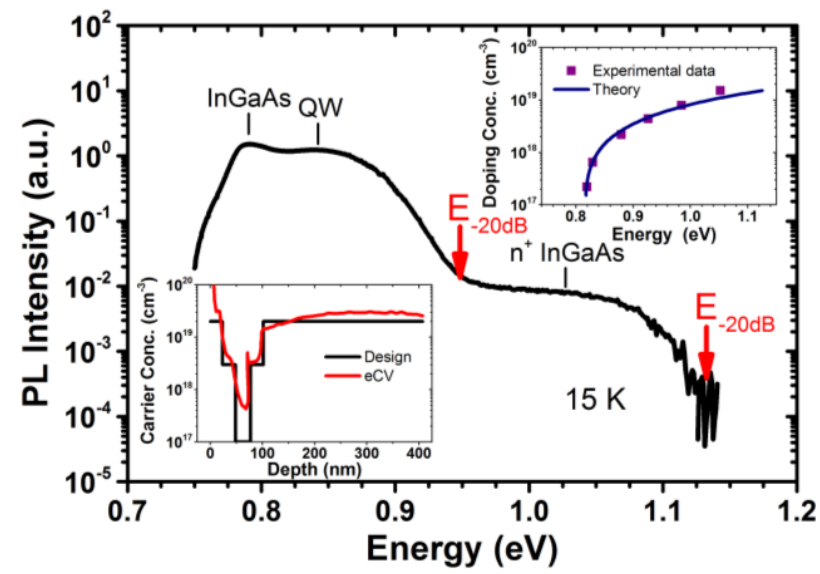

Fig. 6 Low temperature PL spectrum of the RTD structure on a logarithmic scale. The top right inset shows the PL calibration curve and bottom left inset shows the measured free-electron concentration as a function of depth using eCV profiling.

impurity concentrations.

\subsection{Well-Width Dependent Luminescence Characteristics}

PL spectroscopy was performed on RTD structures with different well widths to investigate the well width dependent luminescence characteristics. Figure 7 (a) shows room temperature PL of structures with well widths of 3.0, 3.5, 4.0, and $4.5 \mathrm{~nm}$. The observed PL emission shift towards higher energies with decreasing QW width is attributed to the increase in quantum confinement, as expected.

Whilst room temperature PL is attractive from a manufacturing perspective with tools being readily available allowing rapid and easy characterisation with no cryogenic cooling required, the PL information from room temperature PL spectra remains limited due to the broadness of the emission linewidth. We highlight the need for low temperature PL measurements to investigate the QW and InGaAs in detail, with low temperature PL depending critically on the quality of the structure in terms of alloy composition uniformity and interface abruptness. Figure 7 (b) shows low temperature PL spectra at $15 \mathrm{~K}$ of the same RTD structures in Fig. 7 (a). The larger measured energy shift at low temperature is attributed to the enhanced quantum confinement effect as a result of the increased electronic band-gaps at low temperature. The inset of Fig. 7 (b) shows the experimentally measured and theoretically calculated e $1 \mathrm{hh} 1 \mathrm{QW}$ peak transition energy using a full Schrödinger solver as a function of well width at $15 \mathrm{~K}$. Good agreement is observed between theoretical and experimental data. As expected, the theoretically calculated transition energy is overestimated for a modelled parabolic band structure.

Aside from XRD, PL spectroscopy is also applied to determine the crystal quality. The PL emission linewidth generally gives a good measure of the material quality with narrow linewidths generally indicating high quality QW material. From analysing the low temperature PL emission 
(a)
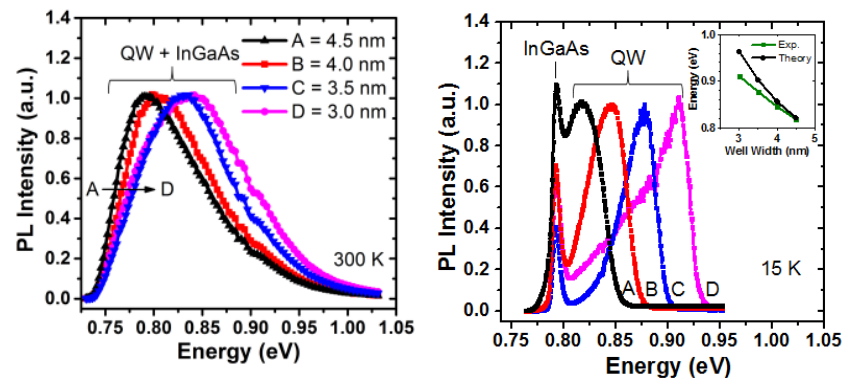

Fig. 7 Normalised PL spectra from RTDs with well widths from 3 to $4.5 \mathrm{~nm}$ measured at (a) $300 \mathrm{~K}$ and (b) $15 \mathrm{~K}$. The inset of (b) shows the experimental and theoretical transition energy as a function of well-width.

linewidth $(\Delta \mathrm{E}=6 \mathrm{meV})$ from the lattice matched undoped InGaAs, variations in alloy composition of $<0.5 \%$ are estimated over the exciton volume. Furthermore, the energetic position of the InGaAs emission line in Fig. 7 (b) confirms lattice matched growth on InP.

Whilst the energetic position of the InGaAs emission line provides information of the ternary alloy composition, the energetic position of QW emission provides information of the average well width, and the emission linewidth provides a good measure of the structural perfection of the QW. In addition to alloy broadening (reduced as compared to lattice-matched ternary due to the higher indium composition), interface disorder and lifetime broadening of the states need to be considered. Alloy broadening is caused by potential fluctuations induced by the random distribution of the different group-III elements (In and Ga) in ternary InGaAs. As the indium concentration in the $\mathrm{QW}$ approaches the binary compound InAs, alloy broadening in the QW is expected to be less pronounced.

We expect alloy compositional fluctuations of less than $1 \%$ in the $\mathrm{In}_{0.80} \mathrm{Ga}_{0.20}$ As QW. Low alloy broadening is required for good device performance to minimise alloy scattering. In 1988, Broekaert et al. reported a high PVCR of 30 at $300 \mathrm{~K}$ for a InGaAs/AlAs/InAs structure with AlAs barriers of ten atomic layers for a peak current density of $6 \mathrm{kA} / \mathrm{cm}^{2}$ [44]. This was achieved by the incorporation of binary InAs in the QW to reduce alloy scattering.

No evidence is found in Fig. 7 (b) of extended ML fluctuations due the lack of strong discrete luminescence peaks in the QW spectra. The lateral extension of interface roughness is therefore expected to be less than the exciton size (few tens of $\mathrm{nm}$ for the in-plane direction [45]). A single emission peak is observed in this case as the individual excitons cannot resolve the fast spatial variation of the lateral extension of single ML growth fluctuations. These excitons are therefore directly probing the interface roughness of the barrier interfaces [46]. We note that ML fluctuations of the barrier thickness are also expected to have a significant role in modifying the linewidth of the transition. Calculation of the electron dwell time gives $60 \mathrm{fs}$ for this structure (1.1 $\mathrm{nm}$ barriers), which corresponds to a lifetime broadening of $3 \mathrm{meV}$. If a $0.8 \mathrm{~nm}$ barrier is considered (a ML fluctuation), this value rises to $6 \mathrm{meV}$. Interface roughness scattering can be reduced by ensuring that the terrace size is much smaller than the electron wavelength such that the electrons experience a smooth barrier due to the fast spatial variation of the interface potential [24]. Similarly, this scattering can also be suppressed when the terrace size is much larger than the electron wavelength for the electrons to experience a smooth surface.

For the QW emission, a monotonic reduction in linewidth is measured from $22 \mathrm{meV}$ to $13 \mathrm{meV}$ at the high energy half width at half maximum (HWHM) side for a reduction in well width from 4.5 to $3.0 \mathrm{~nm}$. The full width at half maximum (FWHM) linewidth is not considered in this case to minimise the interference of the undoped and $\mathrm{n}^{+}$ InGaAs emission in the measurement. The observed reduction in linewidth with decreasing well width is attributed to a combination of alloy and interface disorder. As the penetration of the excitonic wave function in the binary AlAs barriers increases with decreasing well width, less contribution of alloy disorder to the fluctuations of the exciton energy is expected, and the localisation of the electron is reduced in the well [47]. We also note that whilst the influence of interface roughness on the linewidth of infinitely deep QWs increases monotonically for decreasing well-width, and becomes infinite in the limit of zero-width limit; the interface roughness effect for QWs of finite depth reaches a maximum and then decreases to zero in the zero-width limit due to the increasing penetration of the excitonic wave function into the barriers. As the localisation effect of the electron is reduced in the thin $\mathrm{QW}$, the electron dwell time is consequently reduced, which is effective for high frequency performance [48].

\section{Conclusions}

We reported on PL characterisation as a rapid, low-cost, and non-destructive tool for growth monitoring and optimisation of high current density InGaAs/AlAs/InP high current density RTD for THz applications. We investigated the origin of the PL emission in detail using eCV as a selective etch process, combined with a spatially resolved PL line scan technique. We assigned luminescence to the $\mathrm{QW}$, undoped InGaAs buffer layer, and $\mathrm{n}^{+}$InGaAs emitter/collector/ contact layers. As the $\mathrm{n}^{+} \mathrm{InGaAs}$ emission is dominated by the Moss-Burstein effect, we showed how the absolute freeelectron concentration of the emitter/collector and contact layers in the RTD structure can be measured using the PL method and confirmed results by eCV. Information on the alloy composition and compositional variations was extracted from the InGaAs buffer layer, confirming lattice matched growth and alloy compositional fluctuations of less than $1 \%$. We then explored the well width dependent luminescence characteristics, which provided information about the well width and alloy and interface disorder. 


\section{Acknowledgments}

The authors wish to gratefully acknowledge T. Mukai, D. Ohnishi, D. T. D. Childs and O. Wada for valuable discussions. K.J.P. Jacobs gratefully acknowledges EPSRC for a studentship.

\section{References}

[1] T. Nagatsuma, "Terahertz technologies: present and future," IEICE Electron. Express, vol.8, no.14, pp.1127-1142, 2011.

[2] A. Bergner, U. Heugen, E. Bründermann, G. Schwaab, M. Havenith, D.R. Chamberlin, and E.E. Haller, "New p-Ge THz laser spectrometer for the study of solutions: $\mathrm{THz}$ absorption spectroscopy of water," Review of Scientific Instruments, vol.76, no.6, p.063110, 063110-5, June 2005.

[3] B.S. Williams, "Terahertz quantum-cascade lasers," Nat. Photonics, vol.1, pp.517-525, 2007. doi:10.1038/nphoton.2007.166

[4] K. Kawase, J. Shikata, and H. Ito, "Terahertz wave parametric source,” J. Phys. D: Appl. Phys., vol.35, no.3, pp.R1-R14, 2002.

[5] H. Ito, F. Nakajima, T. Furuta, and T. Ishibashi, "Continuous THz-wave generation using antenna-integrated uni-travelling-carrier photodiodes," Semicond. Sci. Technol., vol.20, no.7, pp.S191-S198, 2005.

[6] J. Nishizawa, P. Płotka, T. Kurabayashi, and H. Makabe, "706-GHz GaAs CW fundamental-mode TUNNETT diodes fabricated with molecular layer epitaxy," Phys. Status Solidi C, vol.5, no.9, pp.2802-2804, 2008.

[7] H. Eisele, "480 GHz oscillator with an InP Gunn device," Electron. Lett., vol.46, no.6, pp.422-423, 2010.

[8] V. Radisic, X.B. Mei, W.R. Deal, W. Yoshida, P.H. Liu, J. Uyeda, M. Barsky, L. Samoska, A. Fung, T. Gaier, and R. Lai, "Demonstration of sub-millimetre wave fundamental oscillators using 35-nm InP HEMT technology," IEEE Microw. Compon. Lett., vol.17, no.3, pp.223-225, 2007.

[9] M. Asada and S. Suzuki, "Terahertz oscillators using electron devices - an approach with resonant tunneling diodes," IEICE Electron. Express, vol.8, no.14, pp.1110-1126, 2011.

[10] T. Kleine-Ostmann and T. Nagatsuma, "A review on terahertz communications research,” J. Infrared, Millim. Terahertz Waves, vol.32, no.2, pp.143-171, 2011.

[11] T. Nagatsuma, H. Nishii, and T. Ikeo, "Terahertz imaging based on optical coherence tomography [Invited]," Photon. Res., vol.2, no.4, pp.B64-B69, 2014.

[12] M. Tonouchi, "Cutting-edge terahertz technology," Nature Photon., vol.1, no.2, pp.97-105, 2007.

[13] K.B. Cooper, R.J. Dengler, N. Llombart, A. Talukdera, A.V. Panangadana, C.S. Peay, and P.H. Siegel, "Fast, high-resolution terahertz radar imaging at 25 meters," Proc. SPIE, vol.7671, 2010.

[14] S. Cherry, "Edholm's law of bandwidth," IEEE Spectr., vol.41, no.7, pp.58-60, 2004.

[15] T. Mukai, M. Kawamura, T. Takada, T. Nagatsuma, Tech. Dig. Optical Terahertz Science and Technology Meeting, MF42, Santa Barbara, 2011.

[16] S. Suzuki and M. Asada, "Terahertz oscillators and receivers using electron devices for high-capacity wireless communication," Proc. SPIE, vol.9483, 948309, 2015

[17] H. Sugiyama, H. Yokoyama, A. Teranishi, S. Suzuki, and M. Asada, "Extremely High Peak Current Density of over $1 \times 10^{6} \mathrm{~A} / \mathrm{cm}^{2}$ in InPbased InGaAs/AlAs Resonant Tunneling Diodes Grown by Metalorganic Vapor-phase Epitaxy,” Jpn. J. Appl. Phys., vol.49, no.5, p.051201, 2010 .

[18] T.C.L.G. Sollner, P.E. Tannenwald, D.D. Peck, and W.D. Goodhue, "Quantum Well Oscillators," Appl. Phys. Lett., vol.45, no.12, p.1319, 1984.
[19] K. Murata, K. Sano, T. Akeyoshi, N. Shimizu, E. Sano, M. Yamamoto, and T. Ishibashi, "An optoelectronic clock recovery circuit using a resonant tunneling diode and a uni-traveling-carrier photodiode," IEICE Trans. Electron., vol.E82-C, no.8, pp.1494-1501, 1999.

[20] B. Romeira, J.M.L. Figueiredo, C.N. Ironside, A.E. Kelly, and T.J. Slight, "Optical control of a resonant tunneling diode microwave-photonic oscillator," IEEE Photon. Technol. Lett., vol.22, no.21, pp.1610-1612, 2010.

[21] M. Missous, M.J. Kelly, and J. Sexton, "Extremely Uniform Tunnel Barriers for Low-Cost Device Manufacture," IEEE Electron Device Lett., vol.36, no.6, pp.543-545, 2015.

[22] H. Sugiyama, A. Teranishi, S. Suzuki, and M. Asada, "High-uniformity InP-based resonant tunneling diode wafers with peak current density of over $6 \times 10^{5} \mathrm{~A} / \mathrm{cm}^{2}$ grown by metal-organic vapor-phase epitaxy," Journal of Crystal Growth, vol.336, no.1, pp.24-28, 2011.

[23] P. Blood, "Capacitance-voltage profiling and the characterisation of III-V semiconductors using electrolyte barriers," Semicond. Sci. Technol., vol.1, no.1, pp.7-27, 1986.

[24] P. Roblin, R.C. Potter, and A. Fathimulla, "Interface roughness scattering in AlAs/InGaAs resonant tunneling diodes with an InAs subwell," J. Appl. Phys., vol.79, no.5, p.2502, 1996.

[25] V.A. Wilkinson, M.J. Kelly, and M. Carr, "Tunnel devices are not yet manufacturable," Semiconductor Sci. Technol., vol.12, no.1, pp.91-99, 1997.

[26] R.K. Hayden, A.E. Gunnaes, M. Missous, R. Khan, M.J. Kelly, and M.J. Goringe, "Ex situ re-calibration method for low-cost precision epitaxial growth of heterostructure devices," Semicond. Sci. Technol., vol.17, no.2, pp.135-140, 2002.

[27] P.J. Dean, "Photoluminescence as a diagnostic of semiconductors," Progress in Crystal Growth and Characterization, vol.5, Issues 1-2, pp.89-174, 1982.

[28] C.J.L. Moore and C.J. Miner, "A spatially resolved spectrally resolved photoluminescence mapping system," Journal of Crystal Growth, vol.103, Issues 1-4, pp.21-27, 1990.

[29] Y. Zhang, M. Guan, X. Liu, and Y. Zeng, "Dependence of the electrical and optical properties on growth interruption in AlAs/In0.53Ga0.47As/InAs resonant tunneling diodes," Nanoscale Res. Lett., vol.6, no.1, p.603, 2011.

[30] W.Z. Shen, S.C. Shen, Y. Chang, W.G. Tang, Y. Lu, and A.Z. Li, "Photoluminescence studies of InAs/InGaAs/AlAs strained single quantum well structures," Appl. Phys. Lett., vol.68, no.1, p.78, 1996.

[31] K.J.P. Jacobs, B.J. Stevens, T. Mukai, D. Ohnishi, and R.A. Hogg, "Non-destructive mapping of doping and structural composition of MOVPE-grown high current density resonant tunnelling diodes through photoluminescence spectroscopy," Journal of Crystal Growth, vol.418, pp.102-110, 2015.

[32] J.F. Young, B.M. Wood, G.C. Aers, R.L.S. Devine, H.C. Liu, D. Landheer, M. Buchanan, A.J. SpringThorpe, and P. Mandeville, "Determination of Charge Accumulation and Its Characteristic Time in Double-Barrier Resonant Tunneling Structures Using SteadyState Photoluminescence," Superlattices and Microstructures, vol.5, p.411, 1989; Phys. Rev. Letters, vol.60, no.20, pp.2085-2088, 1988.

[33] P.D. Buckle, P. Dawson, C.Y. Kuo, A.H. Roberts, W.S. Truscott, M. Lynch, and M. Missous, "Charge accumulation in GaAs/AlGaAs triple barrier resonant tunneling structures," J. Appl. Phys., vol.83, no.2, p.882, 1998 .

[34] M.S. Skolnick, D.G. Hayes, P.E. Simmonds, A.W. Higgs, G.W. Smith, H.J. Hutchinson, C.R. Whitehouse, L. Eaves, M. Henini, O.H. Hughes, M.L. Leadbeater, and D.P. Halliday, "Electronic processes in double-barrier resonant-tunneling structures studied by photoluminescence spectroscopy in zero and finite magnetic fields," Phys. Rev. B, vol.41, no.15, pp.10754-10766, 1990.

[35] H.V.A. Galeti, H.B. de Carvalho, M.J.S.P. Brasil, Y.G. Gobato, V. Lopez-Richard, G.E. Marques, M. Henini, and G. Hill, "Role of X valley on the dynamics of electron transport through a GaAs/AlAs double-barrier structure," Phys. Rev. B, vol.78, no.16, p.165309, 
2008.

[36] H. Tews, R. Neumann, and R.D. Schnell, "Growth and characterization of double barrier resonant tunnelling diodes," Journal of Crystal Growth, vol.107, no.1-4, pp.947-951, 1991.

[37] K.K. Smith, "Photoluminescence of Semiconductor Materials," Thin Solid Films, vol.84, no.2, pp.171-182, Oct. 1981.

[38] V.A. Vilkotskii, D.S. Domanevskii, R.D. Kakanakov, V.V. Krasovskii, and V.D. Tkarchev, "Burstein-Moss Effect and Near-Band-Edge Luminescence Spectrum of Highly Doped Indium Arsenide," Phys. Status Solidi B, vol.91, no.1, pp.71-81, 1979.

[39] J. De-Sheng, Y. Makita, K. Ploog, and H.J. Queisser, "Electrical properties and photoluminescence of Te-doped GaAs grown by molecular beam epitaxy," Journal of Applied Physics, vol.53, no.2, p.999, 1982.

[40] W.K. Metzger, M.W. Wanlass, R.J. Ellingson, R.K. Ahrenkiel, and J.J. Carapella, "Auger recombination in low-band-gap n-type InGaAs," Applied Physics Letters, vol.79, no.20, pp.3272-3274, 2001.

[41] R.K. Ahrenkiel, R. Ellingson, S. Johnston, and M. Wanlass, "Recombination lifetime of In $0.53 \mathrm{Ga} 0.47 \mathrm{As}$ as a function of doping density," Applied Physics Letters, vol.72, no.26, pp.3470-3472, 1998.

[42] S. Steiger, M. Povolotskyi, H.-H. Park, T. Kubis, and G. Klimeck, "Nemo5: a parallel multiscale nanoelectronics modeling tool," IEEE Trans. Nanotechnol., vol.10, no.6, pp.1464-1474, 2011.

[43] M. Tajima, "Determination of Boron and Phosphorus Concentration in Silicon by Photoluminescence Analysis," Appl. Phys. Lett., vol.32, no.11, p.719-721, June 1978.

[44] T.P.E. Broekaert, W. Lee, and C.G. Fonstad, "Pseudomorphic In $0.53 \mathrm{Ga} 0.47 \mathrm{As} / \mathrm{AlAs} / \mathrm{InAs}$ resonant tunneling diodes with peakto-valley current ratios of 30 at room temperature," Applied Physics Letters, vol.53, no.16, p.1545, 1988.

[45] M. Grundmann and D. Bimberg, "Anisotropy elects on excitonic properties in realistic quantum wells," Phys. Rev. B, vol.35, no.18, pp.13486-13489, 1988.

[46] M.A. Herman, D. Bimberg, and J. Christen, "Heterointerfaces in quantum wells and epitaxial growth processes: Evaluation by luminescence techniques," Journal of Applied Physics, vol.70, R1, 1991.

[47] A. Patanè, A. Polimeni, M. Capizzi, and F. Martelli, "Linewidth analysis of the photoluminescence of InxGa1-xAs/GaAs quantum wells ( $\mathrm{x}=0.09,0.18,1.0)$," Phys. Rev. B, vol.52, no.4, pp.2784-2788, 1995.

[48] H. Kanaya, S. Suzuki, and M. Asada, "Terahertz oscillation of resonant tunneling diodes with deep and thin quantum wells," IEICE Electron. Express, vol.10, no.18, p.20130501, 2013.

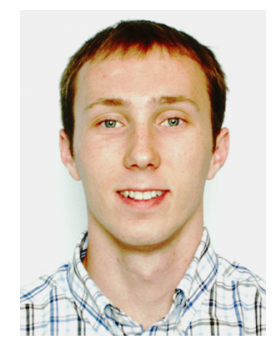

Kristof J. P. Jacobs received the M.Sc. degree in Electrical and Electronic Engineering from the University of Sheffield in 2011. Since joining the University of Sheffield, he has been involved in research on characterisation of III-V compound semiconductors grown by MOVPE, and their device application. His current research focuses on the development of high current density resonant tunnelling diode terahertz emitters.

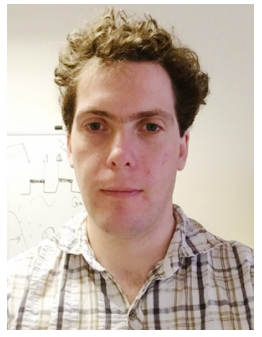

Benjamin J. Stevens was awarded his $\mathrm{PhD}$ in 2010 from the University of Sheffield on advanced GaAs based lasers. During his PhD he was awarded a Japanese Society for the Promotion of Science summer fellowship, which he spent in the Asakawa group in Tsukuba, Japan learning selective area MBE. He worked at the National Centre for III-V Technologies, Sheffield, UK for 6 years from 2009 where he did MOVPE growth of optoelectronic structures. Since 2015 he has worked in the Hogg group in Sheffield UK on the regrowth of photonic crystal surface emitting lasers and the optimisation of RTD epitaxy.

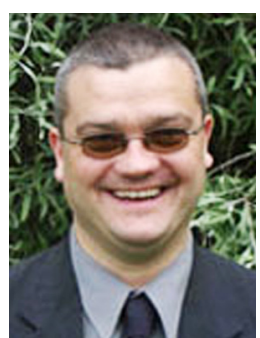

Richard A. Hogg received the Ph.D. degree in physics in 1995 from the University of Sheffield, Sheffield, U.K. For two years, he was a Postdoctoral Research Fellow in NTT Basic Research Laboratories, Atsugi, Japan. He was then awarded an EU-Japan fellowship as a Visiting Researcher in Professor Arakawa's Laboratory, University of Tokyo. For three years, he was in Toshiba Research Europe's Cambridge Laboratory. In 2000, he joined Agilent Technologies Fibre-Optic Component Operation, Ipswich, U.K. In 2003, he joined the Department of Electronic and Electrical Engineering, the University of Sheffield, where he is currently a Professor of semiconductor devices. His current research interests include developing the understanding of device physics and engineering, fabrication technologies, and applications of various semiconductor laser, amplifier, superluminescent diode, and resonant tunnelling devices. 